\title{
Construction and Practice of Undergraduate Teaching Quality Assurance System Based on International Engineering Education Concept
}

\author{
Yong Yang, Jingliang Jiang, Yuanlin Guan, Ning Cui, Chuanwang Song \\ School of Mechanical and Automotive Engineering, Qingdao University of Technology, Qingdao, China \\ Email: yyong901@163.com
}

How to cite this paper: Yang, Y., Jiang, J. L., Guan, Y. L., Cui, N., \& Song, C. W. (2020). Construction and Practice of Undergraduate Teaching Quality Assurance System Based on International Engineering Education Concept. Open Journal of Social Sciences, 8, 255-262.

https://doi.org/10.4236/jss.2020.812019

Received: November 19, 2020

Accepted: December 19, 2020

Published: December 22, 2020

Copyright $\odot 2020$ by author(s) and Scientific Research Publishing Inc. This work is licensed under the Creative Commons Attribution-NonCommercial International License (CC BY-NC 4.0). http://creativecommons.org/licenses/by-nc/4.0/

\begin{abstract}
Aimed at the problems such as old talents training concept, single assessment and lack of long-term continuous improvement mechanism, the undergraduate teaching quality assurance system was constructed and practised. Several crucial aspects such as the quality control of teaching process, the assessment mechanism for curriculum aim and graduation requirements, the teaching quality feedback mechanism, as well as the continuous improvement mechanism were explored. Based on the above research, the undergraduate teaching quality assurance system is formed, which is easy to operate and contains the OBE international advanced concept. Practical application shows that the teaching quality assurance system constructed in this paper can improve our teaching quality effectively.
\end{abstract}

\section{Keywords}

Undergraduate Education, Teaching Quality and Guarantee System, Continuous Improvement, OBE

\section{Introduction}

The development of higher education has shifted from quantity expansion to quality improvement. The comprehensive revitalization of undergraduate education will be the core task of the development of higher education in the new era. Quality development has become the key to the development of the new era. In recent years, the ministry of education has successively promulgated relevant policies and opinions to guide the construction of the teaching quality assurance system (Li, Hei, \& Wang, 2020; Liu, 2016).

Colleges and universities at home and abroad attach great importance to the 
construction of teaching quality assurance system and have made a lot of explorations. In terms of connotation research, Chen Yukun and other experts and scholars believe that the teaching quality assurance system is an internal quality management system covering the whole process of talent training established by universities to improve the teaching quality (Chen, 2004). Li Zhiyi et al. believe that the teaching quality assurance system is an organic whole with clear responsibility, division of labor and clear rights and responsibilities aiming at ensuring and improving the teaching quality (Li, Zhu, \& Liu, 2013). In terms of the research on the composition of the teaching quality assurance system, Li Hanbang and Song Lixia et al. believe that the undergraduate teaching quality assurance system consists of the macro decision control system, the data collection system, the data processing system, the evaluation and diagnosis system and the feedback system (Li \& Song, 2004). In terms of the existing problems in the teaching quality assurance system and the solutions, Sun Fenglin pointed out a series of problems in the teaching quality assurance system in colleges and universities in China, including the lack of scientific evaluation indicators, the existence of passive evaluation objects, and the undiversified evaluation methods (Sun, 2007).

Although some progress has been made, it mainly focuses on the theoretical aspects and has little effect on the practical application. At present, the main problems or disadvantages of the undergraduate teaching quality assurance system include the old talents training concept, single assessment and lack of long-term continuous improvement mechanism. To solve the above problems and disadvantages, the undergraduate teaching quality guarantee system was constructed and practised based on international engineering education OBE concept.

\section{Overall Framework}

Based on the concept of international engineering education (OBE), an overall framework has been carried out in all aspects, from talent training mode to continuous improvement, focusing on high quality professional quality standard and "evaluation of learning" teaching process quality control, objective situation with graduation requirements for output evaluation mechanism. The overall framework was shown in Figure 1.

\section{Key Links of Guarantee System}

\section{1) High quality teaching system}

The high quality undergraduate course teaching system is the necessary premise and foundation of the teaching quality assurance system. In lead of the thought of international engineering education professional certification, based on the concept of international engineering education professional certification, on the basis of education management, system theory, cybernetics and other related theory, research and constructed based on the "project driving training, competition platform culture fusion, fusion culture education, science and education, 


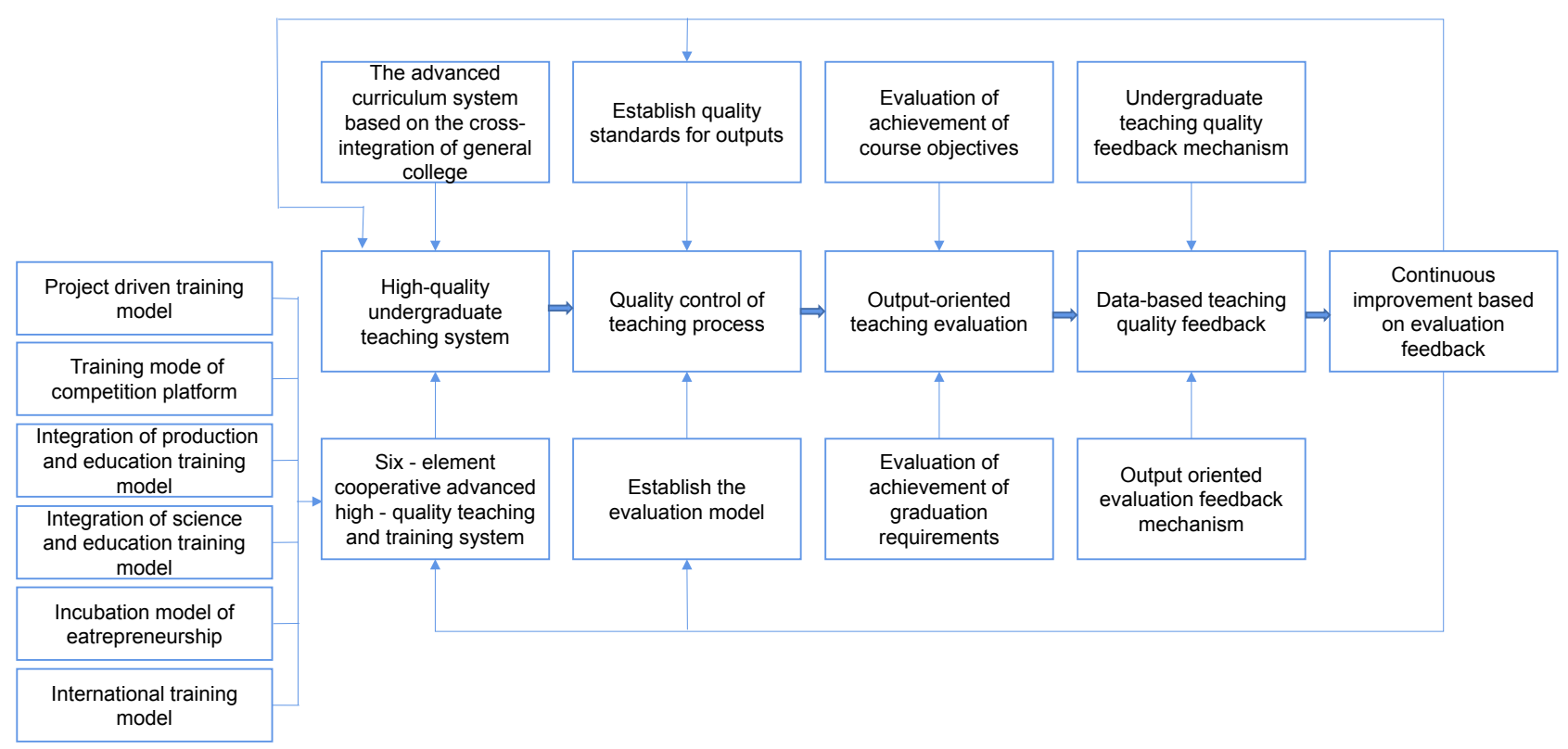

Figure 1. Overall framework.

business incubation cultivation, internationalization" of the "six elements of collaborative and progressive" high quality teaching system, and connotation of the system is shown in Figure 2.

\section{2) Teaching process quality control}

Teaching process quality control is an important link of the teaching quality assurance system. Based on the concept of professional certification, it constructs and practices the output-oriented professional quality standard and "evaluation" mode. Analyses the shortage of the existing teaching process quality monitoring, the concept of professional certification and teaching monitoring actual unifies, established focused on the professional quality of the output oriented concept, will be the main teaching standard export (students) are associated with graduation requirements, in the training objectives, graduation requirements, course system, teaching syllabus and teaching process, the main links such as to make clear quality standards related to graduation requirements; This paper makes a systematic study of the deficiencies of the existing "teaching evaluation" mode, taking students as the center, transforms the monitoring of teachers' teaching into the evaluation of students' output effect, and focuses on the construction of the "evaluation" mode and system oriented to the output effect.

3) Evaluation mechanism for the achievement of output-oriented curriculum objectives and graduation requirements

The establishment of an easy to operate and quantifiable evaluation mechanism is another important link in the construction of high-quality undergraduate teaching quality assurance system. Based on the concept of professional certification, this paper systematically studied and practiced the output-oriented evaluation mechanism of course objectives and graduation requirements, and implemented 


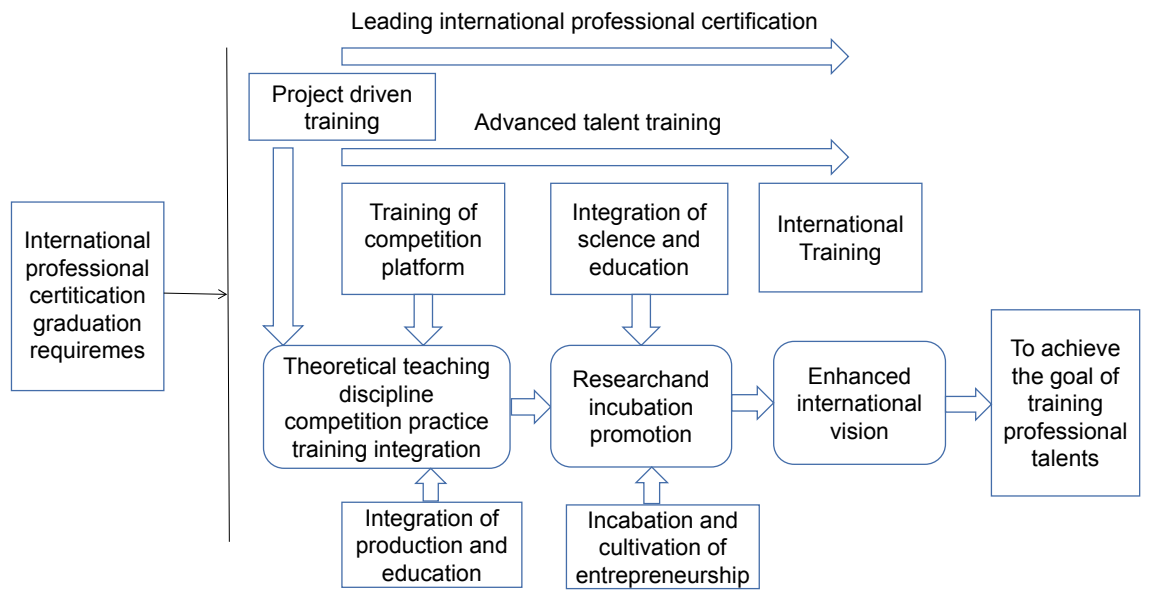

Figure 2. Six elements of collaborative and progressive high quality teaching system.

the professional graduation requirements and course objectives into every course and every teacher through the above mechanism, so as to ensure the achievement of course objectives and the continuous improvement of teaching quality. In terms of the evaluation mechanism of the achievement of course objectives, a scientific and reasonable mathematical model and calculation method of the degree of achievement are developed. Based on the process assessment system and combining with the effective data of students' learning process, the evaluation of the achievement of course objectives is realized through the analysis, comparison and synthesis of a large number of teaching data. Further according to the objective of data, in accordance with the requirements for graduation to reach a degree of mathematical model, divide the curriculum goal comprehensive consideration, correlation index point graduation requirements, formed a graduation requirement agreed upon condition data, the comprehensive analysis of the graduation requirements to achieve data, realize the graduation requirements for students as a whole to achieve situation assessment. The course quality evaluation is the common core of the evaluation mechanism of the achievement of the course objectives and the achievement of the graduation requirements. The output-oriented evaluation mechanism and process are shown in Figure 3.

\section{4) Teaching quality feedback mechanism}

Based on various teaching processes and evaluation "information data", a feedback mechanism of undergraduate teaching quality and an output-oriented evaluation feedback mechanism are established. The main contents include the following four aspects:

a) In-depth construction and improvement of teaching basic state database. Database system adopts open platform design, data collection, management, query, analysis and reporting functions, on the basis of the relevant data of the summary and analysis of the conditions for offering education indicators in daily dynamic monitoring and early warning, regularly every year in the higher education quality monitoring data provided and analysis on the national data 


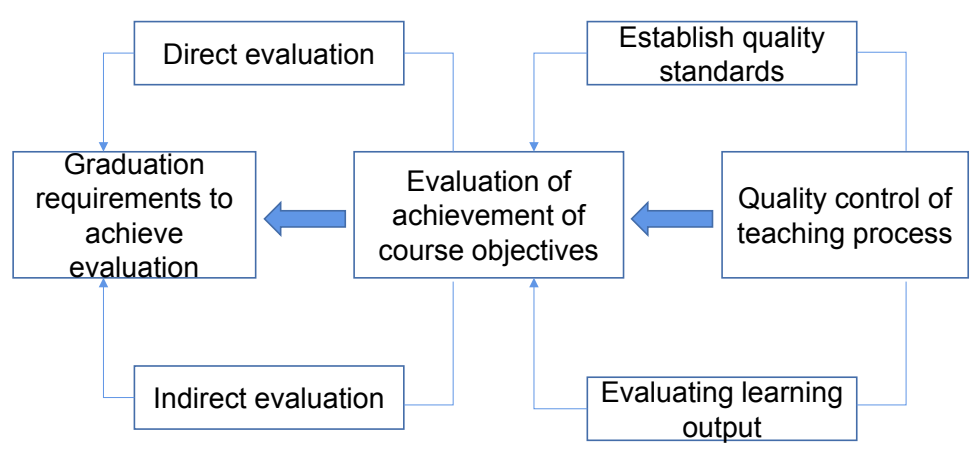

Figure 3. Output-oriented evaluation mechanism and process.

platform, for undergraduate teaching quality guarantee system for data support and decision-making basis.

b) Establish a real-time feedback system for teaching quality. The first is to carry out real-time feedback of daily teaching quality information, and timely feedback of teaching quality monitoring information through teaching inspection notification, student feedback, supervision feedback and other channels, so as to reply to questions and Suggestions raised by teachers and students. The second is to implement the system of "academic discussion and exchange meeting" and "academic early warning" for students, and hold regular student academic discussion meetings to feedback teaching quality data to students. Students who fail more courses than their required academic credits will be given an academic warning.

c) Establish a professional early warning and exit feedback mechanism. It regularly analyzes the enrollment, transfer and employment data of majors, controls the enrollment scale based on data, reduces the enrollment of majors and the amalgamation and cancellation of majors, strengthens the construction of majors from the entrance, and lays a foundation for the improvement of undergraduate teaching quality.

d) Establish an output-oriented evaluation feedback mechanism. On the basis of the evaluation mechanism of the achievement of the output-oriented curriculum objectives and the achievement of the graduation requirements, the evaluation results are obtained according to the curriculum evaluation report, the evaluation results of the curriculum objectives and the evaluation results of the graduation requirements. On the other hand, according to the wishes and needs of off-campus stakeholders, the training objectives are evaluated by alumni discussions, employer surveys and graduate questionnaires, and the evaluation results are timely fed back. The above evaluation results provide data support and decision basis for the construction of undergraduate teaching quality assurance system.

\section{5) Continuous improvement mechanism}

The process of undergraduate teaching quality improvement and guarantee system construction is a process of continuous improvement. This study based on data output oriented evaluation and teaching feedback, further study on the 
teaching quality continuous improvement mechanism, teaching target evaluation results, the result of curriculum evaluation, teaching quality evaluation result and the feedback of graduates tracking for continuous improvement, set up the mechanism of external evaluation, external evaluation and the result was used for training goal with revision of the graduation requirements, graduate degrees. Key contents include continuous improvement of teaching activities to meet the requirements of graduation; Continuous improvement of graduation requirements, in line with the training objectives; Continuous improvement training objectives, in line with the internal and external needs of the three aspects. On this basis, periodic evaluation link and effective continuous improvement mechanism are constructed to form a closed-loop undergraduate teaching quality assurance mechanism and system. The model of continuous improvement adopted is shown in Figure 4.

\section{Practice and Application}

The undergraduate teaching quality guarantee system has been applied in mechanical manufacturing major, setting up the quality control of teaching, curriculum goal and the teaching quality to achieve situation assessment, feedback, continuous improvement, such as process specification file, formed a focus on "output" of the "project teaching", "course evaluation", "continuous improvement" and other system and long running course quality analysis report and other teaching materials.

The practice shows that the level of professional management and construction is constantly improved: 1) making the course keep continuous improvement, to effectively improve the teaching quality; 2) the evaluation and feedback results are used to revise the training program and curriculum system to make it more reasonable and perfect. The evaluation and feedback results of teaching supervision group experts, peer lectures, student evaluation, school evaluation and teacher evaluation are applied to the improvement of main teaching links; 3 )

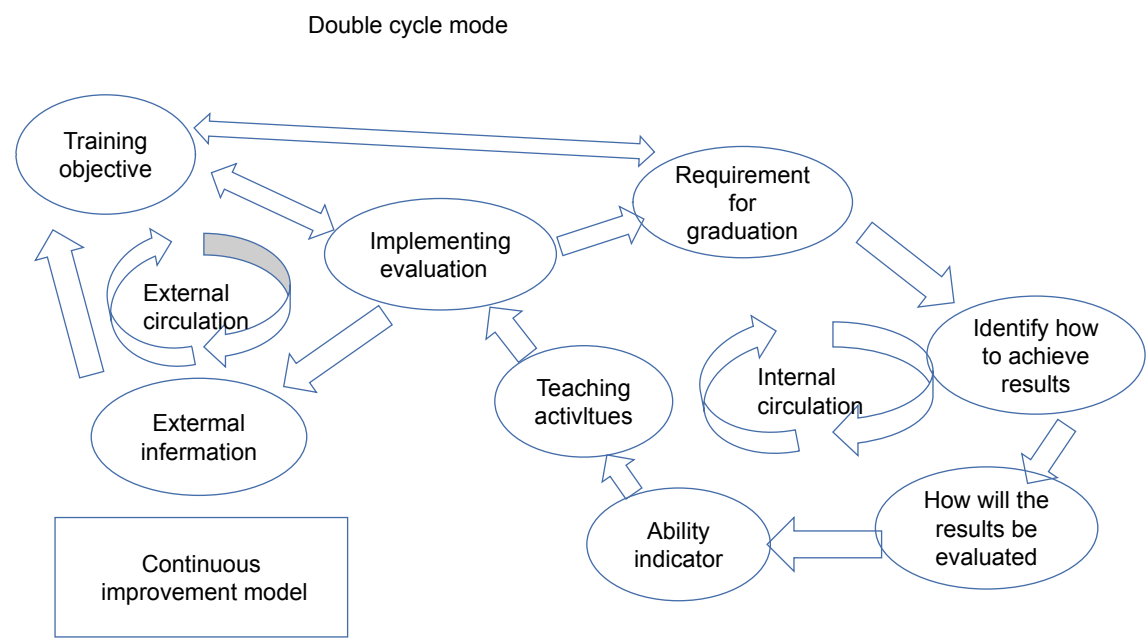

Figure 4. Continuous improvement model based on evaluation and feedback. 
enhancing students' engineering application and innovation ability; 4) promoting the professional school level, the professional development, mechanical design and manufacturing and its automation.

In terms of quality of personnel training, to enhance students' engineering application and innovation ability, the undergraduate scientific research training plan, course contests, apply for a patent, academic papers published, due to the increasing number of graduates employment rate were $97 \%$, nearly three years of grind acceptance rate has remained at more than $25 \%$, to participate in more than 60 national college students' innovative training program projects, won the "challenge cup" national university student extracurricular academic science and technology works competition", "national college students' mechanical innovation design competition" in the national competition, reward 27 items, such as provincial science and technology competition award 120.

At the same time, a survey analysis was made about the teaching effect of the "CDIO project" and the teaching effect questionnaire was shown in Table 1.

As can be seen from the above teaching effect survey, the CDIO project can implement student's ability of practice and innovation, and achieve engineering education training goals.

\section{Conclusion}

1) The undergraduate teaching quality guarantee system was constructed based on the OBE concept. The practical application shows that the guarantee system

Table 1. Teaching effect questionnaire.

\begin{tabular}{|c|c|c|c|c|c|c|c|c|}
\hline \multirow{2}{*}{ No. } & \multirow{2}{*}{ Evaluation index } & \multirow[b]{2}{*}{ Weights } & \multicolumn{6}{|c|}{ Itemized scoring criteria } \\
\hline & & & 10 & 9 & 8 & 7 & 6 & 5 \\
\hline 1 & $\begin{array}{l}\text { "CDIO project" content meets the syllabus } \\
\text { requirements }\end{array}$ & $5 \%$ & $\sqrt{ }$ & & & & & \\
\hline 2 & $\begin{array}{l}\text { "CDIO project" deepens the understanding of the } \\
\text { difficult points of the course }\end{array}$ & $5 \%$ & $\sqrt{ }$ & & & & & \\
\hline 3 & $\begin{array}{l}\text { "CDIO project" focuses on linking theory with practice, } \\
\text { reflecting the development of the discipline }\end{array}$ & $5 \%$ & & $\sqrt{ }$ & & & & \\
\hline 4 & $\begin{array}{l}\text { The "CDIO project" scheme design is reasonable and } \\
\text { feasible }\end{array}$ & $10 \%$ & & $\sqrt{ }$ & & & & \\
\hline 5 & "CDIO project" classroom learning atmosphere is active & $15 \%$ & $\sqrt{ }$ & & & & & \\
\hline 6 & "CDIO project" training hands-on practical ability & $15 \%$ & $\sqrt{ }$ & & & & & \\
\hline 7 & "CDIO project" trains innovative design ability & $15 \%$ & $\sqrt{ }$ & & & & & \\
\hline 8 & $\begin{array}{l}\text { "CDIO project" realizes the application of theoretical } \\
\text { knowledge }\end{array}$ & $15 \%$ & & $\sqrt{ }$ & & & & \\
\hline 9 & "CDIO project" focuses on quality education & $5 \%$ & & $\sqrt{ }$ & & & & \\
\hline 10 & "CDIO project" can improve teaching quality & $10 \%$ & $\sqrt{ }$ & & & & & \\
\hline & Overall rating & 1 & & & 9.65 & & & \\
\hline & Comprehensive level & & & celle & & & & \\
\hline
\end{tabular}

Note: Excellent: 9 - 10; Good: 8 - 8.9; Medium: 7 - 7.9; Pass: 6 - 6.9; Fail: 5 - 5.9. 
can effectively improve the teaching quality and play a significant role in promoting the teaching operation, curriculum construction, talent training and professional certification.

2) The undergraduate teaching quality assurance system can realize the quantification of evaluation indexes and is easier to operate than the traditional teaching quality assurance system, and can effectively solve the problems of "teaching evaluation" thinking and single teaching evaluation form. The output-oriented international engineering education concept is introduced into the construction of teaching quality assurance system, which is more innovative than the traditional teaching quality assurance system based on organizational structure, teaching information feedback and teaching evaluation feedback.

3) The research results have great reference significance for similar domestic universities, providing reference for the reform and innovation of the training mode and teaching management of engineering professionals in relevant universities.

\section{Acknowledgements}

This research was funded by the emerging engineering education research and practice project of the Ministry of Education, grant number E-ZNZZ20201213, the Undergraduate Education Reform Key Project of Shangdong Province, grant number Z2018S020, the Undergraduate Education Reform Breeding Project of Shangdong Province, grant number P2020051, and the Undergraduate Education Reform Major Project of Qingdao University of Technology, grant number 2019072.

\section{Conflicts of Interest}

The authors declare no conflicts of interest regarding the publication of this paper.

\section{References}

Chen, Y. K. (2004). Introduction to Quality Assurance System of Higher Education (pp. 35-40). Beijing: Beijing Normal University Press.

Li, H. B., \& Song, L. X. (2004). Reflections on the Teaching Quality Assurance System in Colleges and Universities. Chinese University Teaching, 2, 52-53.

Li, W., Hei, X. H., \& Wang, L. (2020). Exploration and Practice of Learning Outcome Monitoring and Evaluation Mechanism. Research on Higher Engineering Education, 2, 179-186.

Li, Z. Y., Zhu, Z., \& Liu, Z. J. (2013). Key Points of Design and Implementation of Undergraduate Teaching Audit and Evaluation Program. China University Teaching, 8, 72-77.

Liu, Z. T. (2016). System·Rigidity·Normality: Three Key Words of Internal Quality Assurance System Construction in Higher Education. China Higher Education Research, 9, 12-16.

Sun, F. L. (2007). Discussion on the Establishment of Internal Quality Assurance System in Colleges and Universities. Journal of Shandong Branch of China Women's University, 2, 27-29. 